\title{
Konkret und pragmatisch
}

\author{
Bernhard Bormann
}

In Wohngebieten sind zahlreiche Schlüsselpersonen und Organisationen mit je eigenen Interessen tätig. Eine erfolgreiche Stadtteilarbeit lebt davon, zwischen allen Beteiligten ein funktionierendes Netzwerk zu knüpfen, wie ein Beispiel aus BadenWürttemberg zeigt.

Netzwerke sind ein obligatorisches Muss in der Projektarbeit. Kein Zuschussgeber, der die Vernetzung nicht einfordert, kein Organisationsberater, der nicht die synergetischen Effekte von vernetztem Arbeiten beschwört. Vernetztes Arbeiten soll wahre Wunder bewirken und man hat den Eindruck mit Netzwerkarbeit soll all das aufgefangen werden, was der Sozialstaat nicht mehr leisten kann.

Angesichts eines inflationären Gebrauches des Netzwerkgedankens machte ich den Versuch, aus einer über zehnjährigen Praxiserfahrung in der Stadtteilarbeit, die Tauglichkeit von Netzwerkarbeit auf den Prüfstand zu stellen.

Die Gemeinwesenarbeit auf dem Hardt, dem jüngsten Stadtteil von Schwäbisch Gmünd, nahm seinen Ausgangspunkt im Jahr 1992 kurz nach dem Abzug der amerikanischen Streitkräfte. Der Fall des Eisernen Vorhangs und das Ende des Ost-West-Konflikts brachte es mit sich, dass die in Mutlangen stationierten Pershings abgezogen und damit der komplette US-amerikanische Stützpunkt aufgelöst wurde. Rüstungskonversion der ganz anderen Art war angesagt: was tun mit der »Housing area«, dem Wohngebiet der GI's?

Der Übernacht-Leerstand einer kompletten Siedlung war höchst willkommen in der Zeit der Wohnungsnot Anfang der 1990er Jahre. Die Stadt, bzw. die städtische Wohnungsbaugesellschaft VGW übernahm die Neubesiedlung und sukzessive Sanierung des gesamten Quartiers.

\section{$3 \times 1 / 3=$ gute Bewohnermischung}

Mit dieser Formel - ein Drittel Spätaussiedler, ein Drittel ausländische Familien, ein Drittel Einheimische - sollte von vornherein sichergestellt werden, dass sich auf dem Hardt eine gesunde Bewohnerstruktur entwickelt. Doch die Realität des freien Wohnungsmarktes ließ bald die guten Absichten zur Makulatur werden. Für die wenigen »deutschen« Familien war das multikulturelle Viertel kein Ort, an dem sie sich langfristig niederlassen wollten. Sie zeigten dem Wohngebiet bald den Rücken und zogen weg.

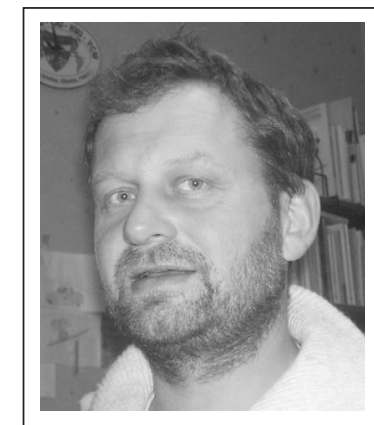

Bernhard Bormann (45) ist Stadtteilkoordinator und Geschäftsführer des Vereins für Jugend-, Familien- und Gemeinwesenarbeit (JuFuN e.V.) in Schwäbisch Gmünd. Das Nachbarschaftszentrum ist ein gemeinwesenorientiertes Stadtteilprojekt in einer ehemaligen amerikanischen Wohnsiedlung, die überwiegend von Migrantenund Spätaussiedlerfamilien bewohnt ist. Neben der Netzwerkarbeit in dieser Siedlung ist Bormann auch in anderen Netzwerken tätig, z. B. Tauschnetz Bumerang und Familienaktives undattraktives Schwäbisch Gmünd. Aktuell entwickelt sich in der Oststadt Schwäbisch Gmünds ein Netzwerkprozess beim Aufbau eines Quartiersmanagements im Rahmen des Stadtteil-Entwicklungsprogamms »Die Soziale Stadt .

Internet http://www.jufun.de

Eine außerordentlich hohe Konzentration von Familien mit Migrationshintergrund (über $90 \%$ ) ist heute ein Faktum, das vielleicht zu bedauern ist, aber auch durch noch so viele Anstrengungen in der Wohnbewirtschaftung (Modernisierungsmaßnahmen) und Steuerung bei der Wohnungsvergabe nicht umkehrbar ist.

Es bleibt die Herausforderung für die Gemeinwesenarbeit, das soziale Leben und Zusammenleben in der Siedlung von über 25 verschiedenen Nationalitäten- und Kulturgruppen so zu organisieren, dass die gegebene »Vitalität der Vielfalt « letztlich auch als Chance von den Bewohnern wahrgenommen wird.

\section{Aus einem Schulterschluss wird ein runder Tisch}

Zurück zu den Anfängen: Die Vergabe der 220 Wohnungen wurde in atemberaubender Zeit von drei Monaten abgewickelt. Bei den Verantwortlichen in der Stadt, der Wohnungsbaugesellschaft und einigen engagierten Bürgern und Einrichtungen - die katholische Kirchengemeinde St. Peter und Paul vorneweg - war schnell die Einsicht gewonnen, dass das Viertel nicht sich selbst überlassen werden darf. Wollte man nicht gänzlich die Kontrolle über die sich abzeichnenden negativen Entwicklungen verlieren, dann brauchte es ein konzertiertes Vorgehen. $\rightarrow$ 
In einer Stadtteilanalyse unter Anleitung von Prof. Dr. Wolfgang Hinte vom Institut für Stadtteilbezogene Soziale Arbeit und Beratung der Universität Essen wurde eine ehrliche Bestandsaufnahme vorgenommen. Die Maßnahmen, die im Jahr 1992 empfohlen wurden, waren und sind die Blaupause für einen Prozess, der bis ins Jahr 2006 andauert:

- Abstimmung der im Stadtteil tätigen Professionellen: Einrichtung eines »Runden Tisches « aller beteiligten Mitarbeiter, Abstimmung thematischer, unter den Institutionen abgesprochener Aktivitäten, bessere Koordination materieller und kommunikativer Hilfen

- Organisation von Bürgerinteressen und Initiierung von Selbsthilfeaktivitäten: Initiierung eines Mieterrates, Selbsthilfegruppen von Alleinerziehenden und Sozialhilfeempfängern, ehrenamtliches Engagement im Bereich der Kinder- und Jugendarbeit, Aktivitäten im Wohnumfeld sowie Mitarbeit bei der Gestaltung von Fluren und Wohnungen

- Imagewerbung für den Stadtteil: Werbeaktionen sowohl nach innen als auch nach außen zur Unterstützung des Rufs des Stadtteils als ein Gebiet, in dem es sich zu wohnen lohnt

Mittelfristig empfahl Wolfgang Hinte die Einrichtung einer Planstelle zur Verstärkung der Kinder- und Jugendarbeit als auch zur Initiierung und Unterstützung weiterer

\section{Was erreicht werden konnte}

Als große Errungenschaften im Bemühen um eine nachhaltige Entwicklung auf dem Hardt können im Rückblick gesehen werden:

- Einrichtung einer wöchentlichen Sprechstunde des Sozialamtes im Wohngebiet

- Etablierung einer Jugendsozialarbeit in der Siedlung durch die Schaffung einer Sozialpädagogen-Stelle und eines Anerkennungspraktikanten sowie der Einrichtung eines offenen Jugendtreffs: Ferienspiele, Kinderfeste, Sport-Events, erlebnispädagogische Maßnahmen sowie soziales Kompetenztraining in Kooperation mit der Hauptschule sind feste Bestandteile der Jugendarbeit im Wohngebiet.

- jährlich stattfindende Stadtteilfeste (Hardtfest, Weihnachtsfeier)

- Initiierung einer Mietervertretung. Nicht zuletzt durch deren Wirken konnte eine umfangreiche Sanierungsund Modernisierungsmaßnahme angestoßen werden.

- Aufbau des Familien- und Nachbarschaftszentrums

- Bau eines Gemeinschaftshauses mit den Bewohnern

- weitverzweigte Stadtviertelarbeit ausgehend vom Familien- und Nachbarschaftszentrum: Gemeinschaftsaktivitäten, Sauberkeitsaktionen, Pflanzaktionen, Sprachbildung, Computerkurse, Kinderangebote, Leseförderung für Grundschüler, »Computerstüble« (für Erwachsene und Kinder), Computerkeller für Jugendliche

- Sozialberatung für die Bewohner in allen Alltagsbelangen und muttersprachliche Familienbildung und Familienbegleitung durch türkisch und russisch-sprechende Mitarbeiterinnen

- Jüngste Aktionen sind die Schaffung von Treffpunkten im Freien und die Renovierung einer Multisportanlage, um mehr Freizeitorte für Cliquenjugendliche zu schaffen. Damit sollen die »vagabundierenden « Jugendlichen erreicht werden, die sich nicht durch einen offenen Jugendtreff ansprechen lassen.

Dies war der Ausgangspunkt zur Gründung eines »Großen Runden Tisches Hardt «. Alle Akteure, die in direkter und indirekter Weise für die sozialen Belange auf dem Hardt involviert sind, treffen sich seit 1992 in regelmäßigen Abständen (zwei- bis viermal im Jahr) um über die Entwicklungen der Hardt-Siedlung zu beraten und vor allem um geeignete Maßnahmen in die Wege zu leiten.

Da Gemeinwesenarbeit immer auch systemischer Ansatz bedeutet, versteht sich von selbst, dass ein unterstützendes Netzwerk fachübergreifend alle Akteure an einen Tisch bringt, die zur Gesamtentwicklung etwas beitragen können. Dabei gibt es zwei Kategorien von Akteuren:

- Die unmittelbar im Wohngebiet tätigen sozialen Einrichtungen: Kirchengemeinden, Kindergärten, Kinderhort, Schule, Jugendtreff, Nachbarschaftstreff, Mietervertretung, Gemeinschaftsunterkunft für Asylbewerber

- kommunale Akteure, Behörden und sonstige Träger der Sozialarbeit: Stadt/Sozialamt, Wohnungsbaugesellschaft, staatliches Schulamt, Polizei, Landkreis Ostalb (Sozialdezernat - Jugendamt - Amt für Integration und Versorgung), Caritas, Volkshochschule

\section{Chancen und Grenzen von Netzwerkarbeit}

Die Aufzählung vermittelt den Eindruck, dass Netzwerkarbeit automatisch solche Erfolge hervorbringen kann. Davor sei aber allemal gewarnt. Dennoch gibt es drei Grunderfahrungen aus zwölf Jahren Netzwerkarbeit, die sich auf andere Kontexte übertragen lassen:

1. Gemeinwesenorientierte Netzwerke sollten von bürgerschaftlicher Seite getragen sein und eine starke Unterstützung durch die Kommune haben: Erst das Zusammenspiel beider politischen Bereiche bewirkt, dass Erkenntnisse aus der Sozialanalyse auch in konkrete Veränderung münden. Netzwerke sind »vorparlamentarische« Strukturen. In der Regel müssen Vorschläge den Weg über die Verwaltung und die Gremien (Ausschüsse, Gemeinderat) zur Entscheidung geführt werden. Wenn in Netzwerken die Verwaltung von Anbeginn mit eingebunden ist, dann sind schon viele Hürden auf dem Weg zur Umsetzung ausgeräumt. Von großer Bedeutung ist 
auch die Bekanntheit des Netzwerkes in der Öffentlichkeit und eine (formelle) Anerkennung durch die Verwaltung.

2 Netzwerke leisten keine konzeptionelle Arbeit: 2 Netzwerke haben den Nachteil, dass sie aufgrund der Größe und der heterogenen Zusammensetzung nicht in der Lage sind, größere Lösungsansätze zu entwickeln. Daher ist die Frage zu klären, an welchen Orten vorgedacht und Projekte entwickelt werden. Weitergehende Impulse kamen am Beispiel des »Großen Runden Tisches Hardt « durch Studien- und Reflexionstage mit fachlicher Begleitung von außen. Oder die Impulse wurden durch einzelne Akteure ins Netzwerk hineingetragen und fanden dort Resonanz und Unterstützung.

3. Netzwerke leben von konkreten pragmatischen Aufgaben: Weit gesteckte Ziele (z. B. eine positive Stadtteilentwicklung, die jeder befürworten wird), reichen als Selbstverständnis eines Netzwerkes nicht aus. Auch die »Verzahnung der personellen und institutionellen Ressourcen « ist noch zu abstrakt und wird keine Dynamik auslösen. Notwendig sind konkrete Projekte, für die es sich lohnt, alle Kräfte auszurichten. Dies sind auf dem Hardt die Stadtteilfeste, der Bau eines Nachbarschaftstreffpunktes und aktuell das Zusammenwachsen des alten und neuen Hardts als Konsequenz aus dem 50-jährigen Siedlungsjubiläum, das im Jahr 2004 ein neues Wir-Gefühl geweckt hatte.

\section{Netze, mit denen man Fische fängt}

Neue Initiativen und Projekte in der heutigen Zeit ins Leben zu rufen, ist ein schwieriges Unterfangen. Der soziale und kulturelle Sektor gerät immer mehr in die Defensive. Freiwilligenleistungen werden von Gemeinden, Städten, Landkreisen, Land und Bund angesichts einer immer prekäreren Finanzlage der öffentlichen Hand systematisch zurückgefahren. So scheint es um des Erfolges willen immer notwendiger zu werden, Ideen und Visionen nicht in kleinen Zirkeln oder kooperationsresistenten Trägerbunkern zu entwickeln, sondern im Netzwerk.

Man sollte die multiplizierende Kraft von Netzwerkarbeit nicht unterschätzen. Die Chance der Kooperation über den Tellerrand hinaus, aber braucht ein neues vernetztes Denken.

Daher funktionieren Netzwerke nur mit Akteuren, die den gemeinsamen »Mehrwert « von kooperativem Handeln verstanden haben und dies in ihrer beruflichen Praxis umsetzen und (er-)leben wollen.

Ein Letztes: Das Prinzip Nachhaltigkeit betrifft genauso die Netzwerkarbeit. Nur wenn personelle und finanzielle Ressourcen dauerhaft bereitgestellt werden, also nicht als Brandherd-Löschmittel zur Eindämmung schlimmer sozialer Fehlentwicklungen betrachtet werden, kann eine langfristige präventive soziale Entwicklungsarbeit geleistet werden.

Und diese zahlt sich allemal aus.

\section{Erfolgsfaktoren in der Netzwerkarbeit}

In dem Projekt »Nachhaltiges Wirtschaften: Möglichkeiten und Grenzen neuer Nutzungsstrategien - regionale Ansätze« des Bundesforschungsministeriums stellt die »Wissenschaftliche Begleitforschung, Moderation und Coaching regionaler Netze auf dem Gebiet neuer Nutzungsstrategien « fünf bedeutsame Bedingungen in der Zusammenarbeit vor Ort heraus:

- Sinn und Potenziale - d. h. die Verfügbarkeit eines gemeinsamen Verständnisses unter den Netzwerkpartner bezüglich Nutzen und Entwicklungszielen des Netzwerks sowie die Ausstattung des Netzwerks mit ausreichend Potenzialen und deren Nutzung

- Beziehung, Soziales und Identität - d. h. Schaffung einer Kultur des Vertrauens, der Gegenseitigkeit und Kooperation sowie einer Gemeinsamkeit in der Identität und Arbeitsteilung

- Information und Kommunikation d. h. Bereitstellung einer Plattform und Gewährleistung einer Transparenz durch abgestimmte Informationsflüsse

- Management und Organisation - d. h. unter Beachtung der Basisprinzipien der Netzwerkarbeit die Führung, Steuerung, Moderation und das Controlling derselben

- Umwelt-und Rahmenbedingungen $d$. h. die Beachtung der aus systemischer Sicht externen Einflüsse, denen das Netzwerk ausgesetzt ist

fx - Institut für zukunftsfähiges Wirtschaften e. V., Marienstraße 19/20, 10117 Berlin, E-Mail info@fhochx.de, Internet http://www.fhochx.de 